\title{
Oesophageal carcinoma presenting with a synchronous asymptomatic colon carcinoma
}

\author{
Alok Gupta, Bharat Chauhan, \\ V Rangarajan ${ }^{1}$, Saral Desai ${ }^{2}$, \\ Vanita Noronha,
}

Kumar Prabhash

Departments of Medical Oncology,

${ }^{1}$ Nuclear Medicine and ${ }^{2}$ Pathology,

Tata Memorial Hospital, Mumbai,

Maharashtra, India

\author{
Address for correspondence: \\ Dr. Vanita Noronha, \\ Department of Medical Oncology, \\ Tata Memorial Hospital, Mumbai, \\ Maharashtra, India. \\ E-mail: vanita.noronha@gmail.com
}

\author{
A B S T R A C T
}

The advancement in diagnostic techniques has resulted in increased incidence of occult second primary in cancer patients. Here, we report a case of symptomatic oesophageal carcinoma and synchronous asymptomatic colon carcinoma diagnosed through Positron Emission Tomography-Computed Tomography imaging.

Key words: Multiple primary malignant neoplasms, oesophageal carcinoma, synchronous colon carcinoma

\section{INTRODUCTION}

Multiple primary malignant neoplasms (MPMN) were first described by Billroth in 1889. ${ }^{[1]}$ Since then, numerous studies and reports have appeared on the subject. The prevalence of MPMN was reported between $0.734 \%$ and $11.7 \%$ in a review of $1,104,269$ cancer patients. ${ }^{[2]}$ Modern diagnostic procedures contribute to the detection of synchronous occult tumors which may have formerly been overlooked. The present case is one such example where PET-CT performed for staging evaluation of a symptomatic oesophageal carcinoma led to the diagnosis of a synchronous asymptomatic colon carcinoma and also changed the neoadjuvant chemotherapy regimen used.

\section{CASE REPORT}

A 58-year female, known case of hypertension and type-2 diabetes mellitus, with no history of addictions presented with history of dysphagia to solid food and weight loss of $5 \mathrm{~kg}$ over last 2 months. There was no history of dysphagia to liquids, hoarseness of voice, throat pain, epigastric

\begin{tabular}{|l|l|}
\hline \multicolumn{3}{|c|}{ Access this article online } \\
\hline Quick Response Code: & Website: \\
\hline & www.jmpo.org \\
\hline
\end{tabular}

or retrosternal pain, chronic cough, breathlessness, or fever. There was no family history of cancer. Contrast Enhanced Computed Tomography (CECT) thorax revealed thickening of lower third oesophagus with growth extending into gastro-oesophageal (GE) junction and proximal stomach. Enlarged gastrohepatic lymph nodes were present with no significant mediastinal lymphadenopathy. Upper gastrointestinal endoscopy revealed an ulceroproliferative lesion from $33 \mathrm{~cm}$ to $38 \mathrm{~cm}$ with involvement of GE junction and extending to cardia. Biopsy of the lesion was obtained and histopathological examination was suggestive of a poorly differentiated adenocarcinoma [Figure 1]. As a part of staging workup, PET-CT was done which revealed metabolically active disease at lower third oesophagus and GE junction along with celiac lymphadenopathy. It also showed increased uptake in splenic flexure of colon [Figure 2]. Diagnostic colonoscopy revealed an ulceroproliferative lesion at splenic flexure with mild luminal compromise. Histopathology was suggestive of a moderately differentiated adenocarcinoma [Figure 3]. As the two tumors were at two separate geographical sites and were histopathologically different, being poorly differentiated adenocarcinoma for oesophagus and moderately differentiated adenocarcinoma for colon, diagnosis of carcinoma oesophagus with synchronous colon carcinoma was made. Patient was administered four cycles of FOLFIRI regimen (5-fluorouracil, leucovorin, and irinotecan) as neoadjuvant chemotherapy. Repeat PET-CT showed partial metabolic and morphological regression of circumferential thickening in both 


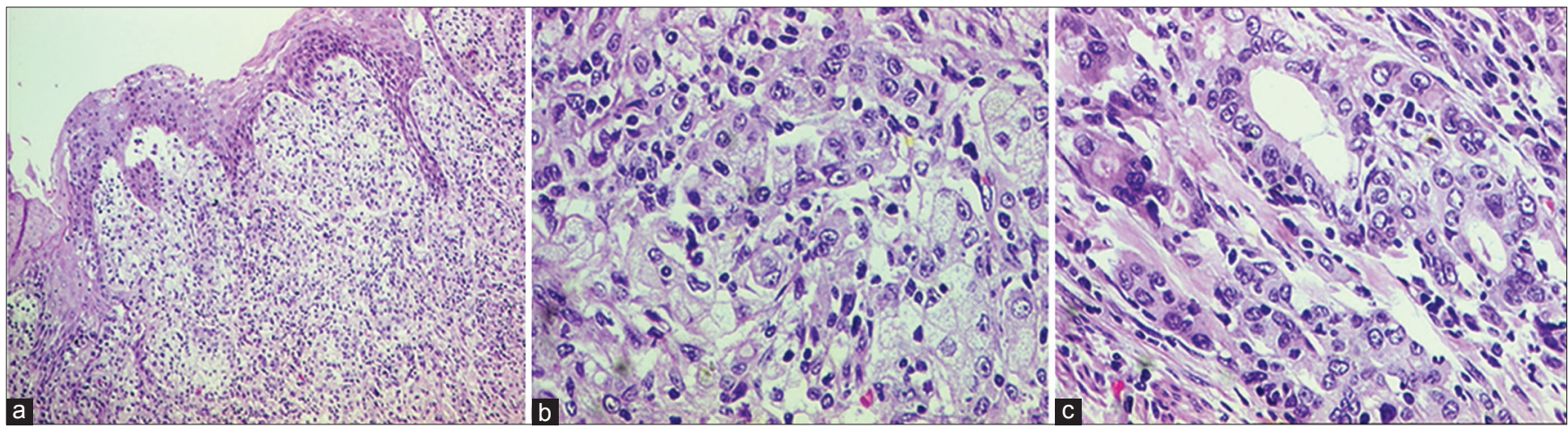

Figure 1: The oesophagus showed groups and cords of carcinoma cells ( $a$ and b), with focal gland formation (c)

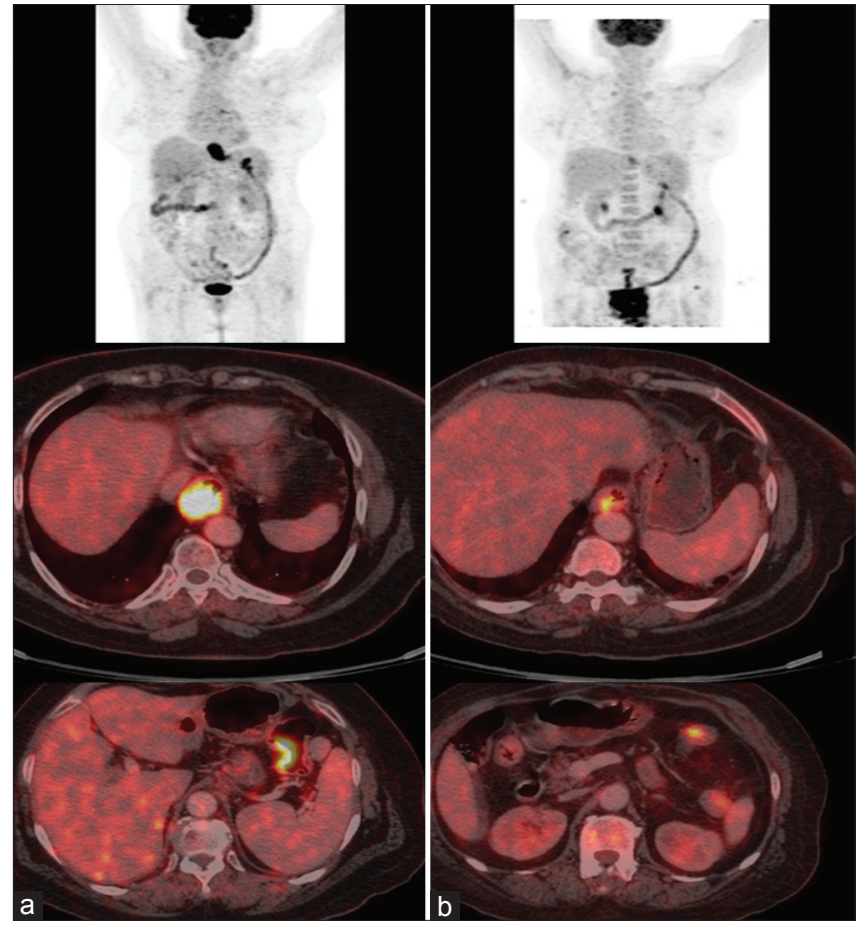

Figure 2: Positron Emission Tomography- Computed Tomography PET-CT imaging showing active disease in lower third oesophagus (thick arrow) and splenic flexure of colon (thin arrow) (a) pre and (b) post neoadjuvant chemotherapy

lower third oesophagus and splenic flexure of colon. Oesophageal wall thickness at the site of lesion decreased from $2.8 \mathrm{~cm}$ to $1.2 \mathrm{~cm}$, while that at the splenic flexure of colon was reduced to no obvious mucosal thickening in the repeat scan. Upper gastrointestinal endoscopy showed residual nodularity and infiltration from $33 \mathrm{~cm}$ to $37 \mathrm{~cm}$ with involvement of GE junction and extending to cardia. Endoscopic ultrasonography revealed that the growth involved adventitia but was free from surrounding structures (T3) with no mediastinal or celiac lymph nodes (N0). The patient underwent transhiatal esophagogastrectomy and left hemicolectomy (R0 resection). Histopathological report reconfirmed the oesophageal lesion to be poorly differentiated adenocarcinoma. No lymphovascular or perineural invasion was seen and proximal and distal margins were free of tumor. Upper, middle, and lower paraoesophageal, hepatic, and splenic nodes were negative for metastases; however, a single lymph node along the lesser curvature showed metastasis. Left hemicolectomy specimen showed moderately differentiated adenocarcinoma with tumor infiltrating the muscularis propria but not through it. No lymphovascular or perineural invasion was seen and proximal and distal margins were free of tumor. Regional lymph nodes were negative for metastasis. Final diagnoses were oesophageal adenocarcinoma T3N1M0 and colon carcinoma T2N0M0. The patient received adjuvant chemotherapy with four cycles of FOLFIRI regimen. The patient was then kept on regular follow-up with repeat Carcino Embryonic Antigen CEA levels; upper and lower gastrointestinal endoscopy and scans planned at every 3 months for 6 months. After 3 months of follow-up, the patient remains asymptomatic and negative for disease relapse on investigations.

\section{DISCUSSION}

MPMN can occur at any age. However, the patients with MPMN tend to be older than those with a single primary malignant neoplasm because the incidence of malignancy increases with age. ${ }^{[3,4]}$ MPMN diagnosed at an earlier age are likely to be associated with family history or genetic predisposition of MPMN. ${ }^{[5]}$ Synchronous carcinomas are those diagnosed at the same time or within a 6-month period after the diagnosis of the initial cancer. Metachronous carcinomas are different cancers that develop over 6 months after the diagnosis of the primary cancer, usually after the treatment of the primary lesion. ${ }^{[6]}$ The reported incidence of synchronous malignancy in patients with MPMN varies between $19 \%$ and $34 \% .^{[3,7,8]}$ Most of the malignancies with MPMNs involve the gastrointestinal, respiratory, and genitourinary systems. ${ }^{[9]}$ With advances in diagnostic techniques and treatment modalities, the number of patients identified with MPMN has been increasing. The phenomenon of MPMN might 


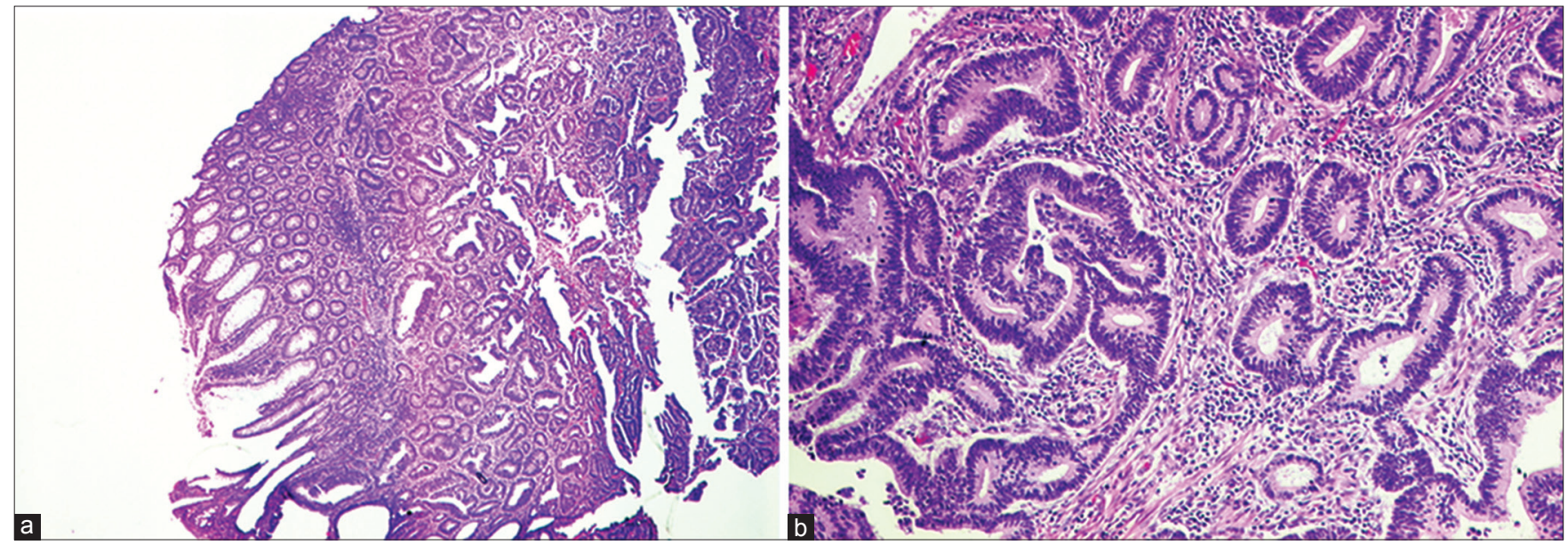

Figure 3: The tumor in the colon was a typical colonic type of adenocarcinoma ( $a$ and $b$ )

reflect underlying genetic or immunological defects in the patient, treatment-related damage, or environmental exposure to carcinogens. ${ }^{[10]}$ Carcinogenesis in different organs subjected to the same carcinogens and promoting factors has led to the concept of a multicentric pathogenic process termed "field cancerization." "[11] Molecular studies also support the alternative theory of a common clonal origin, especially in MPMN of head and neck regions. ${ }^{[1]}$ Besides this, various host genetic factors could significantly modulate the risk of developing tobacco-related cancer including those related to various isoforms of glutathione $S$-transferases, cytochrome P450, $N$-acetyl transferase, and sulfotransferase enzymes. ${ }^{[12]}$

There have been reports that indicated an association between Barrett's metaplasia or adenocarcinoma of the oesophagus and colon carcinoma. ${ }^{[13]}$ However, a subsequent case-control study from Finland showed that Barrett's oesophagus is not associated with an increased risk of colonic carcinoma, negating the need for routine colonoscopic surveillance in patients with Barrett's oesophagus. ${ }^{[14]}$

Patients with colorectal cancer have extraintestinal primary cancers 1.4 times more often than expected. Although reports about the relationship between colon cancer and synchronous oesophageal carcinoma have been published, ${ }^{[15]}$ there were few reports in which colonic carcinoma was asymptomatic and was diagnosed incidentally as a second primary neoplasm. It has been proposed that environmental factors such as alcohol, smoking and diet, and genetic factors regulating increased expression of the cyclooxygenase-2 enzyme are central to the predisposition to both oesophageal and colon cancers. ${ }^{[16]}$ Since this enzyme is inhibited by non-steroidal anti-inflammatory drugs such as aspirin and sulindac, these drugs hold promise as cancer chemopreventive agents in both oesophageal and colon cancers.
PET-CT imaging in oesophageal carcinoma leads to detection of unsuspected metastasis in 15\% of patients and leads to alteration of intended treatment plan in $20 \%$ of patients. It is also of value in evaluating response to chemotherapy and radiotherapy. ${ }^{[17]}$ This report underscores the importance of performing a PET-CT in a patient with oesophageal carcinoma as this not only led to the diagnosis of synchronous colonic carcinoma but also changed the treatment plan. Treatment of synchronous oesophageal and colonic cancers is directed at both the primaries simultaneously. Simultaneous resection of oesophageal and extra oesophageal neoplasms can be safely performed. ${ }^{[18]}$ The standard chemotherapy regimens for oesophageal cancer are cisplatin and 5-fluorouracil (5-FU). However, oesophageal cancer associated with colon cancer has been successfully treated with combination chemotherapy of FOLFOX (5-fluorouracil, leucovorin, and oxaliplatin) and concurrent radiotherapy. ${ }^{[15]}$ In our case, FOLFIRI regimen was used as neoadjuvant chemotherapy which showed good response at both the tumor sites as suggested by PET-CT imaging.

Through this report, we want to emphasize that (a) the possibility of synchronous primary neoplasm should be kept in mind and actively sought for while evaluating a case of oesophageal carcinoma. (b) PET-CT is a useful imaging modality in finding asymptomatic second neoplasm in a case of oesophageal carcinoma. (c) FOLFIRI regimen can be considered in the treatment of oesophageal carcinoma presenting with synchronous colon carcinoma.

\section{REFERENCES}

1. Billroth T. Die Allgemeine Chirurgische Pathologie and Therapie. In: Reimer G, Editor. 51 Vorlesungen-Ein Handbuch fur Studierende and Artze. 14. Vol. 8. xx, Berlin: Auflage; 1889. p. 712.

2. Demandante CG, Troyer DA, Miles TP. Multiple primary malignant neoplasms: Case report and a comprehensive review of the literature. Am J Clin Oncol 2003;26:79-83. 
3. Aydiner A, Karadeniz A, Uygun K, Tas S, Tas F, Disci R, et al. Multiple primary neoplasms at a single institution: Differences between synchronous and metachronous neoplasms. Am J Clin Oncol 2000;23:364-70.

4. Vyas JJ, Deshpande RK, Sharma S, Desai PB. Multiple primary cancers in Indian population: Metachronous and synchronous lesions. J Surg Oncol 1983;23:239-49.

5. Luciani A, Balducci L. Multiple primary malignancies. Semin Oncol 2004;31:264-73.

6. Kilciksiz S, Gokce T, Baloglu A, Calli A, Kaynak C, Kilic B, et al. Characteristics of synchronous- and metachronous-type multiple primary neoplasms: A study of hospital-based cancer registry in Turkey. Clin Genitourin Cancer 2007;5:438-45.

7. Crocetti E, Arniani S, Buiatti E. Synchronous and metachronous diagnosis of multiple primary cancers. Tumori 1998;84:9-13.

8. Koutsopoulos AV, Dambaki KI, Datseris G, Giannikaki E, Froudarakis M, Stathopoulos E. A novel combination of multiple primary carcinomas: Urinary bladder transitional cell carcinoma, prostate adenocarcinoma and small cell lung carcinoma: Report of a case and review of the literature. World J Surg Oncol 2005;3:51.

9. Artac M, Bozcuk H, Ozdogan M, Demiral AN, Sarper A, Samur $\mathrm{M}$, et al. Different clinical features of primary and secondary tumors in patients with multiple malignancies. Tumori 2005;91:317-20.

10. Hemminki K, Boffetta P. Multiple primary cancers as clues to environmental and heritable causes of cancer and mechanisms of carcinogenesis. IARC Sci Publ 2004;157:289-97.

11. Carey TE. Field cancerization: Are multiple primary cancers monoclonal or polyclonal? Ann Med 1996;28:183-8.

12. Kotnis A, Kannan S, Sarin R, Mulherkar R. Case-control study and meta-analysis of SULT1A1 Arg213His polymorphism for gene, ethnicity and environment interaction for cancer risk. $\mathrm{Br} \mathrm{J}$ Cancer 2008;99:1340-7.

13. Sontag SJ, Schnell TG, Chejfec G, O'Connell S, Stanley MM, Best W, et al. Barrett's oesophagus and colonic tumours. Lancet 1985;1:946-9.

14. Laitakari R, Laippala $P$, Isolauri J. Barrett's oesophagus is not a risk factor for colonic neoplasia: A case-control study. Ann Med 1995;27:499-502.

15. Watanabe $Y$, Tsutsui $M$, Takeda S, Yoshino S, Oka M. A case of esophageal cancer associated with colon cancer successfully treated with combination chemotherapy of FOLFOX and concurrent radiotherapy. Gan To Kagaku Ryoho 2009;36:2439-41.

16. Morgan G, Vainio H. Barrett's oesophagus, oesophageal cancer and colon cancer: An explanation of the association and cancer chemopreventive potential of non-steroidal anti-inflammatory drugs. Eur J Cancer Prev 1998;7:195-9.

17. Weber WA, Ott K, Becker K, Dittler HJ, Helmberger H, Avril NE, et al. Prediction of response to preoperative chemotherapy in adenocarcinomas of the esophagogastric junction by metabolic imaging. J Clin Oncol 2001;19:3058-65.

18. Suzuki S, Nishimaki T, Suzuki T, Kanda T, Nakagawa S, Hatakeyama K. Outcomes of simultaneous resection of synchronous esophageal and extraesophageal carcinomas. J Am Coll Surg 2002;195:23-9.

How to cite this article: Gupta A, Chauhan B, Rangarajan V, Desai $\mathrm{S}$, Noronha V, Prabhash K. Oesophageal carcinoma presenting with a synchronous asymptomatic colon carcinoma. Indian J Med Paediatr Oncol 2013;34:117-20.

Source of Support: Nil, Conflict of Interest: None declared. 